\section{Síndrome disentérico en un preescolar con colitis ulcerada}

\section{Dysenteric syndrome in a preschool with ulcerated colitis.}

Ridaura-Sanz $\mathrm{C}^{1}$, Reyes-Cadena $\mathrm{A}^{2}$, Campos-Rivera $\mathrm{T}^{3}$

\section{RESUMEN DE HISTORIA CLÍNICA}

Niño preescolar de 2 años 3 meses de edad, producto de la primera gesta. Madre de 19 años, ama de casa, escolaridad secundaria; padre de 20 años, soldador, preparatoria incompleta, aparentemente sanos. Se negó consanguinidad y endogamia. Nació a las 40 semanas de gestación, por vía vaginal, con una calificación Apgar de 8/9. Su peso al nacimiento fue de $2.8 \mathrm{~kg}$ y midió $51 \mathrm{~cm}$. El tamiz metabólico y auditivo se reportaron normales. Se alimentó al seno materno exclusivo hasta los 6 meses de edad, con alimentación complementaria (papillas de frutas y verduras) a los 6 meses, fue integrado a la dieta familiar a los 12 meses. Su desarrollo psicomotor fue referido como normal. Al momento del ingreso con marcha completa y lenguaje al 100\%.

Medicina preventiva. No mostró cartilla de inmunizaciones, refiriendo falta de una dosis de pentavalente. Habitaba en zona urbana, en una casa construida con materiales perdurables, con agua, luz y drenaje intradomiciliario. Se refirió adecuada ventilación, dos habitaciones donde habitaban tres personas. Disponía de área para la preparación y consumo de alimentos. Baño con cambio de ropa interior y exterior diario, aseo bucal 1 vez al día. Convivía con un perro no vacunado. COMBE negado. Exantemáticas: interrogadas y negadas.

Antecedentes personales patológicos: refirió un traumatismo craneoencefálico leve en región temporal 6 meses antes sin complicaciones.
${ }^{1}$ Médico Anatomopatólogo. Departamento de Patología.

${ }^{2}$ Médico Pediatra. Adscrito a Consulta Externa.

${ }^{3}$ Jefe del Servicio de Parasitología.

Instituto Nacional de Pediatría.

Recibido: 22 de noviembre del 2017

Aceptado: 22 de noviembre del 2017

Correspondencia

Dra. Cecilia Ridaura Sanz

cridaura@gmail.com

Este artículo debe citarse como Ridaura-Sanz C, Reyes-Cadena A, Campos-Rivera T. Síndrome disentérico en un preescolar con colitis ulcerada. Acta Pediatr Mex. 2018;39(1):60-71. 
Su padecimiento inició aproximadamente una semana previa a su ingreso con rinorrea hialina, evacuaciones disminuidas en consistencia sin moco ni sangre, (4 al día) y vómitos postprandiales no cuantificados. Dos días después, se agregó fiebre de $38.3^{\circ} \mathrm{C}$ administrándose metamizol a dosis no referidas. Por persistir con sintomatología acudió con médico particular el cual inició tratamiento con meclozina, trimetoprim con sulfametoxazol, diyodohidroxiquinoleína, furazolidona, bromuro de homatropina, caolín, pectina, no se especificaron las dosis que se administraron durante 3 días sin mejoría, por lo que consultó con un segundo médico que lo trató con dimenhidrinato, dosis no especificada. Se refirió además candidiasis oral y faringoamigdalitis, por lo que se le aplicó antibiótico intramuscular no especificado. Evolucionó a los dos días siguientes con somnolencia, astenia, adinamia, distensión abdominal, dolor abdominal en región periumbilical y posteriormente en marco cólico; baja ingesta de alimentos, fiebre hasta $39^{\circ} \mathrm{C}$ y vómitos de contenido gastrointestinal en 4 ocasiones. Se refirió última evacuación un día antes de su ingreso con restos de moco y sangre, acudiendo a clínica particular donde se evidenció perímetro abdominal aumentado y dos vómitos biliares. Fue referido a nuestro instituto para valoración y manejo.

Ingresó paciente activo, reactivo, irritable, poco cooperador, con dolor abdominal en reposo. Somatometría: peso $10.5 \mathrm{~kg}$ percentil 3 (P3), talla $84 \mathrm{~cm}$ (P10), peso para talla P50, perímetro abdominal (PA) $47 \mathrm{~cm}$. Signos vitales al ingreso: frecuencia cardiaca (FC) 156 latidos por minuto, frecuencia respiratoria (FR) 28 respiraciones por minuto, tensión arterial (TA) 92/57 mmHg.

A la exploración física se refirió con palidez de tegumentos y mucosas semihidratadas, normocéfalo, ojos ligeramente hundidos, narinas permeables, cuello sin adenomegalias, cardiopulmonar sin compromiso, abdomen globoso por distensión importante, hiperbaralgesia, dolor intenso a la palpación generalizada, timpánico a la percusión, peristalsis ausente. Extremidades íntegras, eutróficas, sin alteraciones evidentes, pulsos distales palpables, Ilenado capilar de dos segundos.

En la radiografía de abdomen se documentó dilatación importante de asas intestinales, asas fijas y niveles hidroaéreos.

Fue valorado por el servicio de cirugía pediátrica. Se colocó sonda orogástrica que drenó material en pozos de café. Presentó una evacuación con moco y sangre en el pañal. Paciente se consideró con abdomen agudo y datos sugestivos de perforación intestinal. Se consideró que requería manejo quirúrgico urgente.

En la valoración prequirúrgica se refirió en malas condiciones generales, en ayuno, con sonda nasogástrica a derivación. El perímetro abdominal aumentó a razón de $1 \mathrm{~cm} / \mathrm{h}$. Se dejó manejo con soluciones de base más el $10 \%$, aumentar goteo a $85 \mathrm{~mL} / \mathrm{h}$, reposición por pérdidas al $100 \%$ con solución fisiológica, metronidazol $30 \mathrm{mg} / \mathrm{kg} / \mathrm{d}$ cada 8 h y ceftriaxona $100 \mathrm{mg} / \mathrm{kg} / \mathrm{d}$ cada 12 h, omeprazol $1 \mathrm{mg} / \mathrm{kg} / \mathrm{do}$, vitamina K $0.03 \mathrm{mg} / \mathrm{kg} /$ do dosis única intravenosa (Cuadro 1).

El mismo día de su ingresó se realizó laparotomía exploratoria (LAPE) + apendicectomía + descompresión retrógrada con los siguientes hallazgos: yeyuno e íleon dilatados, plastrón en fosa ilíaca derecha, olor fétido. Apéndice cecal purulento, con perforación y necrosis en la punta. Se colocó drenaje tipo Penrose en fosa ilíaca derecha dirigido a corredera parieto cólica. Se mantuvo hemodinámicamente estable en periodo transanestésico, se transfundieron $244 \mathrm{~mL}$ de concentrado eritrocitario, requirió de albúmina al 5\%, $20 \mathrm{~mL}$ y gluconato de calcio. Sangró $80 \mathrm{~mL}$, se reportó balance hídrico positivo $+283 \mathrm{~mL}$. Durante la evolución posquirúrgica inmediata se refirió afebril con inicio de peristalsis y con secreción purulenta a través del 
Cuadro 1. Exámenes de laboratorio de ingreso

\begin{tabular}{|c|c|c|c|c|c|c|c|c|c|c|}
\hline $\mathrm{Hb}$ & Hto & Leuc & Neutr & Linf & Mono & Plaq & TP & TTP & & \\
\hline $\begin{array}{l}10.2 \\
\mathrm{~g} / \mathrm{dL}\end{array}$ & $30 \%$ & $\begin{array}{l}9,100 / \\
\mathrm{mm} 3\end{array}$ & $\begin{array}{c}11 \% \\
(1,100 / \mathrm{mm} 3)\end{array}$ & $\begin{array}{c}17 \% \\
(1,500 \mathrm{~mm} 3)\end{array}$ & $\begin{array}{c}72 \% \\
(6,600 / \mathrm{mm} 3)\end{array}$ & $\begin{array}{c}107,000 / \\
\mathrm{mm} 3\end{array}$ & $\begin{array}{l}28.9^{\prime \prime \prime} \\
26 \% .\end{array}$ & $26 \%$ & & \\
\hline $\mathrm{pH}$ & $\begin{array}{c}\mathrm{PCO} 2 \\
(\mathrm{mmHg})\end{array}$ & $\underset{(\mathrm{mmHg})}{\mathrm{PO2}}$ & $\begin{array}{c}\mathrm{HCO} 3 \\
(\mathrm{mmol} / \mathrm{L})\end{array}$ & EB & AG & $\begin{array}{c}\mathrm{LAC} \\
(\mathrm{mmol} / \mathrm{L})\end{array}$ & & & & \\
\hline 7.51 & 33.5 & 37.3 & 26.4 & 3.4 & 14.6 & 2.4 & & & & \\
\hline $\begin{array}{c}\text { ALB } \\
(\mathrm{md} / \mathrm{dL})\end{array}$ & TAG & $\begin{array}{l}\mathrm{COL} \\
(\mathrm{mg} / \mathrm{dL})\end{array}$ & $\begin{array}{c}\text { HDL } \\
(\mathrm{mg} / \mathrm{dL})\end{array}$ & $\begin{array}{c}\text { LDL } \\
(\mathrm{mg} / \mathrm{dL})\end{array}$ & $\begin{array}{c}\text { VLDL } \\
(\mathrm{mg} / \mathrm{dL})\end{array}$ & $\begin{array}{c}\mathrm{FA} \\
(\mathrm{mg} / \mathrm{dL})\end{array}$ & $\begin{array}{l}\text { DHL } \\
\text { (U/L) }\end{array}$ & $\begin{array}{c}\text { AST } \\
(\mathrm{mg} / \mathrm{dL})\end{array}$ & $\begin{array}{c}\text { ALT } \\
(\mathrm{mg} / \mathrm{dL})\end{array}$ & $\begin{array}{l}\text { GGT } \\
(\mathrm{mg} / \mathrm{dL})\end{array}$ \\
\hline 1.5 & 16 & 86 & 34.2 & 3.8 & 11.6 & 46 & 450 & 29 & 6 & 22 \\
\hline $\begin{array}{c}\mathrm{Na} \\
(\mathrm{mEq} / \mathrm{L})\end{array}$ & $\begin{array}{c}\mathrm{K} \\
(\mathrm{mEq} / \mathrm{L})\end{array}$ & $\underset{(\mathrm{mEq} / \mathrm{L})}{\mathrm{Cl}}$ & $\begin{array}{c}\mathrm{Ca} \\
(\mathrm{mg} / \mathrm{dL})\end{array}$ & $\begin{array}{c}P \\
(\mathbf{m g} / \mathbf{d L})\end{array}$ & $\underset{(\mathrm{mg} / \mathrm{dL})}{\mathrm{Mg}}$ & $\begin{array}{c}\text { Creat } \\
(\mathrm{mg} / \mathrm{dL})\end{array}$ & BUN & $\underset{(\mathrm{mg} / \mathrm{dL})}{\mathrm{Glu}}$ & $\begin{array}{c}\text { Urea } \\
\text { (mg/dL) }\end{array}$ & $\underset{(\mathrm{mg} / \mathrm{dL})}{\mathrm{Au}}$ \\
\hline 132 & 3.5 & 92 & 7.6 & 4 & 1.7 & 0.66 & 16 & 86 & 34.2 & 3.8 \\
\hline
\end{tabular}

drenaje Penrose con material biliar por sonda. Continuó con manejo en ayuno y cobertura antibiótica con ceftriaxona y metronidazol (día 4).

La evaluación posterior se refirió con intolerancia a la ingestión de líquidos con vómitos de contenido gástrico. Continuó con dolor abdominal, así como múltiples picos febriles hasta $38^{\circ} \mathrm{C}$. Se evidenció gasto verdoso y fétido por Penrose, labstix con gasto biliar $+++y$ sangre +++ . Clínicamente en mal estado general, palidez generalizada, taquicárdico (FC de 128-137 por minuto sin fiebre). Se sospechó perforación intestinal.

Al octavo día de su ingreso se practicó segunda LAPE de urgencia con los siguientes hallazgos: cavidad abdominal con abundante materia fecal, una perforación en la totalidad del ciego, otra de $5 \mathrm{~mm}$ en colon ascendente y con una perforación de $5 \mathrm{~cm}$ en sigmoides abarcando recto. Debido a estos hallazgos se decidió realizar colectomía total + ileostomía de 1 boca a $15 \mathrm{~cm}$ de la válvula ileocecal + aseo de cavidad abdominal. Se colocaron drenajes tipo Penrose derecho e izquierdo. Se transfundieron $293 \mathrm{~mL}$ de concentrado eritrocitario y $190 \mathrm{~mL}$ de plasma fresco congelado. Egresó de quirófano hemodinámicamente estable.
Progresó hacia la gravedad con datos de choque séptico, de necrosis en el estoma y en la herida quirúrgica, con deterioro ventilatorio y hemodinámico, descenso de la cuenta leucocitaria y de plaquetas por lo que requirió intubación y ventilación asistida (Cuadro 2).

Un hemocultivo periférico reportó Pseudomonas aeruginosa, por lo que se inició cobertura con carbapenémico para enterobacterias Gram negativas con patrón de resistencia BLEE o Amp C, así como para Pseudomonas spp. (meropenem $20 \mathrm{mg} / \mathrm{kg} / \mathrm{d}$ cada 8 horas), por las características de piel de herida quirúrgica se decidió cubrir $S$. aureus meticilino resistente con glucopéptido (vancomicina $60 \mathrm{mg} / \mathrm{kg} /$ día cada 6 horas). Se inició infusión de hidrocortisona y milrrinona. Se agregó a esquema antibiótico cobertura con piperacilina (300 mg/kg/día cada 6 horas) y fluconazol (12 mg/kg/día cada 24 horas).

En la Unidad de Terapia Intensiva se refirió taquicárdico, hipotenso a pesar de manejo inotrópico vasopresor y de cargas con coloides. Ventilación mecánica en modo asisto-controlado (AC), manejado por presión con presión inspiratoria de $19 \mathrm{~cm} / \mathrm{H} 2$, presión positiva al final de la espiración de $5 \mathrm{~cm} / \mathrm{H} 2 \mathrm{O}$, fracción inspiratoria de oxígeno al $80 \%$. Tuvo hipoglucemias requiriendo 
Cuadro 2. Exámenes de laboratorio (segundo día posquirúrgico)

\begin{tabular}{|c|c|c|c|c|c|c|c|c|c|c|}
\hline $\begin{array}{c}\mathrm{Hb} \\
(\mathrm{g} / \mathrm{d})\end{array}$ & $\begin{array}{l}\text { Hto } \\
(\%)\end{array}$ & $\begin{array}{c}\text { Leuc } \\
\text { (cel/mcL) }\end{array}$ & $\begin{array}{l}\text { Neutr \% } \\
\text { (cel/mcL) }\end{array}$ & $\begin{array}{c}\text { Linf \% } \\
\text { (cel/mcL) }\end{array}$ & $\begin{array}{l}\text { Mono \% } \\
\text { (cel/mcL) }\end{array}$ & $\begin{array}{c}\text { Plaq } \\
\text { (cel/mcL) }\end{array}$ & Tp & TTP & & \\
\hline 10.2 & 30 & $\begin{array}{c}9, \\
100\end{array}$ & $\begin{array}{c}11 \% \\
(1,100)\end{array}$ & $\begin{array}{c}17 \% \\
(1,500)\end{array}$ & $\begin{array}{c}72 \% \\
(6,600)\end{array}$ & $\begin{array}{l}107 \\
000\end{array}$ & $\begin{array}{l}28.9^{\prime \prime} / \\
26 \% .\end{array}$ & $26 \%$ & & \\
\hline $\begin{array}{c}\mathrm{Na} \\
(\mathrm{mEq} / \mathrm{L})\end{array}$ & $\begin{array}{c}\mathrm{K} \\
(\mathrm{mEq} / \mathrm{L})\end{array}$ & $\begin{array}{c}\mathrm{Cl} \\
(\mathrm{mg} / \mathrm{dL})\end{array}$ & $\begin{array}{c}\mathrm{Ca} \\
(\mathrm{mg} / \mathrm{dL})\end{array}$ & $\begin{array}{c}P \\
(\mathrm{mg} / \mathrm{dL})\end{array}$ & $\begin{array}{c}\mathrm{Mg} \\
(\mathrm{mg} / \mathrm{dL})\end{array}$ & $\begin{array}{l}\text { Creat } \\
\text { (mg/dL) }\end{array}$ & BUN & $\begin{array}{c}\text { Glu } \\
\text { (mg/dL) }\end{array}$ & $\begin{array}{c}\text { Urea } \\
\text { (mg/dL) }\end{array}$ & $\mathrm{Au}(\mathrm{mg} / \mathrm{dL})$ \\
\hline 135 & 1.9 & 102 & 9.1 & 2.3 & 2.8 & 0.44 & 2.8 & 57 & & \\
\hline $\mathrm{pH}$ & $\begin{array}{c}\text { PCO2 } \\
(\mathrm{mmHg})\end{array}$ & $\begin{array}{c}\mathrm{PO} 2 \\
(\mathrm{mmHg})\end{array}$ & $\begin{array}{c}\mathrm{HCO} 3 \\
(\mathrm{mmol} / \mathrm{L})\end{array}$ & EB & AG & $\begin{array}{c}\mathrm{LAC} \\
(\mathrm{mmol} / \mathrm{L})\end{array}$ & & & & \\
\hline 7,47 & 34.3 & 85.5 & 24.2 & 0.5 & 9.3 & 4.4 & & & & \\
\hline
\end{tabular}

cargas con solución glucosada y se aumentó aporte de glucosa $/ \mathrm{kg} / \mathrm{min}$. Cursó con hipokalemia por lo que se aumentó aporte en soluciones.

El paciente cursó con choque séptico refractario a aminas, hipotenso a pesar de vasopresor a dosis tope, anuria por más de 8 horas, mal estado de perfusión generalizada. Ventilatorio con tórax restrictivo que requirió parámetros ventilatorios altos secundario a edema agudo pulmonar y sangrado pulmonar activo ocasionando hipoxemia y desaturación hasta 30\%. Gastrointestinal con compromiso abdominal con necrosis del estoma, drenajes de Penrose con abundante gasto fétido. Metabólico cursó con acidosis metabólica, con disminución de bicarbonato hasta $15 \mathrm{mmol} / \mathrm{L}$ a pesar de cargas y aporte de bicarbonato hasta $4 \mathrm{mEq} / \mathrm{kg}$, con datos de sufrimiento tisular, con lactato de $20 \mathrm{mmol} / \mathrm{L}$. Sufrió bradicardia por debajo de 40 latidos por minuto y paro cardiorrespiratorio que no revirtió a las maniobras de reanimación. Falleció a los 11 días de su ingreso y a 3 días de la última intervención quirúrgica. No se autorizó el estudio postmortem.

\section{COMENTARIO CLÍNICO}

\section{Dr. Armando Reyes Cadena}

En el caso de un paciente pediátrico con evacuaciones con sangre se debe sospechar inicialmente que corresponde a un cuadro de enteritis invasiva, lo que conlleva un riesgo de complicaciones graves e incluso fatales. El hecho de aparición de sangre en heces diarreicas es un motivo de alarma para familiares y médicos, por lo que es necesario la detección precoz de su etiología para iniciar una conducta que pueda modificar el curso de ésta. Dentro de los agentes etiológicos a sospechar se encuentran: Shigella, Salmonella, Escherichia coli enteroinvasiva, Campylobacter, Yersinia y Entamoeba histolytica. Entre las causas no infecciosas se podría considerar las malformaciones vasculares del intestino, la diverticulitis y la poliposis colónica. En el presente caso, además de las evacuaciones sanguinolentas, se presentó fiebre y dolor abdominal, lo que orienta más hacia las causas infecciosas. De los agentes etiológicos mencionados la que cursa con apendicitis y colitis perforadas es la Entamoeba histolytica, con estas complicaciones poco frecuentes que condicionan una elevada mortalidad.

La población pediátrica de los países en vías de desarrollo, como México, se encuentra expuesta a una fácil contaminación parasitaria, dadas las condiciones económicas, sociales, culturales e higiénicas en las cuales crece y se desarrolla. La Organización Mundial de la Salud reporta que la Entamoeba histolytica causa aproximadamente 50 millones de casos y 100 mil muertes anualmente. El colon y el hígado son los principales órganos afectados. E. histolytica causa un 
espectro de enfermedad intestinal que incluye infección asintomática, infección no invasiva sintomática, disentería aguda y colitis fulminante con perforación. La colitis amebiana aguda tiene un inicio gradual manifestándose con una historia de 1-2 semanas con dolor abdominal, diarrea y tenesmo. La infección es usualmente autolimitada pero puede recurrir, especialmente en áreas endémicas. El periodo de incubación de la amebiasis intestinal en promedio es de 1-4 semanas.

En cuanto a las manifestaciones clínicas que se observan en la amebiasis, en la edad pediátrica, se encuentran comúnmente: dolor abdominal, fiebre mayor a $38^{\circ} \mathrm{C}$ (hasta en un tercio de los casos), náusea, vómitos, heces sanguinolentas, hiporexia y pérdida de peso; síntomas que en su mayoría presentó el paciente.

El desarrollo de colitis fulminante, ameboma y amebiasis cutánea pueden ocurrir como complicaciones de la amebiasis intestinal. La colitis amebiana fulminante es una complicación rara de la disentería amebiana, ocurre en aproximadamente $0.5 \%$ de los pacientes con infección sintomática; se presenta con un rápido inicio de diarrea sanguinolenta y dolor abdominal grave, con hipersensibilidad al rebote a la palpación y distensión abdominal, fiebre de alto grado. La indicación para realizar cirugía es perforación intestinal, así como persistencia de dolor, distensión e hipersensibilidad al rebote al realizar palpación abdominal.

En base a la sintomatología del paciente y a lo expuesto previamente se debe considerar lo siguiente:

- En el paciente pediátrico con evacuaciones con sangre y moco, así como evolución subaguda, se debe sospechar etiología amebiana.

- En caso de amebiasis intestinal con una evolución no habitual se debe descartar una complicación como colitis tóxica amebiana.

- Realizar una adecuada semiología permite llegar a un probable diagnóstico etiológico evitando así el uso indiscriminado de antibióticos y antiparasitarios.

Sin embargo, llama la atención que esta patología ocurrió en un niño aparentemente sano, bien nutrido y con condiciones ambientales e higiénicas adecuadas. A pesar de las numerosas investigaciones que se han realizado en relación a la interacción huésped parásito, aún no se conoce con exactitud qué es lo que determina la susceptibilidad a desarrollar amebiasis invasora, ya que en la mayoría de los casos la Entamoeba histolytica se comporta como un comensal con infecciones asintomáticas o autolimitadas. Se sugiere que la susceptibilidad a la infección y su desenlace es multifactorial y está influenciada por factores dependientes del hospedero, del parásito y del ambiente.

Este paciente no presentaba los conocidos factores de riesgo que aumentan la susceptibilidad a amebiasis invasora, como son desnutrición, enfermedades crónicas debilitantes o uso continuo de esteroides. Sin embargo, hay otros condicionantes del huésped que desconocemos en este niño como son los factores genéticos, mencionados en la literatura, en particular la asociada con el polimorfismo del receptor de la arginina o la ausencia de inmunoglobulina A (IgA) intestinal que se ha informado en niños enfermos.

Por su parte, el genotipo del parásito también influye en el desenlace de la infección, se han encontrado diferencias del genotipo del parásito en pacientes con diarrea no complicada de aquellos con absceso hepático amebiano.

Las características del microbioma intestinal también han sido implicados en la susceptibili- 
dad a la infección parasitaria y en particular la infección mixta en enfermedad diarreica, en la que se documentan bacterias entero patógenas, parásitos y virus. La E. histolytica se ha encontrado asociada con rotavirus, Aatrovirus, E. Coli y Clostridium perfringes.

Finalmente, cabe el comentario sobre el fracaso al tratamiento, ya que este niño fue tratado con el esquema tradicional: metronidazol; sin embargo, no se obtuvo respuesta. Aunque es indudable la eficacia de muchos de los antiparasitarios actualmente en el mercado, hay que considerar la dosis y el momento del tratamiento. En este caso desconocemos la dosis empleada y si se administró correctamente, lo que hizo posible que la medicación se instalara en etapa tardía, cuando ya la infección se había complicado.

\section{COMENTARIO ANATOMOPATOLÓGICO}

\section{Dra. Cecilia Ridaura Sanz}

Para el estudio anatomopatológico se recibieron dos piezas quirúrgicas: la primera se envió con el diagnóstico clínico de apendicitis aguda perforada y mostraba al apéndice cecal fragmentado en dos porciones, cubierto por material fibrinopurulento o que se extendía al mesoapéndice. Histológicamente se encontró necrosis isquémica transmural con pérdida de la mucosa y con exudado inflamatorio, predominantemente de polimorfonucleares en el peritoneo y vasculitis importante con trombosis arterial. Días después se recibió el producto de colectomía total en dos segmentos: el mayor de $45 \mathrm{~cm}$ de largo incluía íleon terminal, ciego y colon ascendente y el otro, de $15 \mathrm{~cm}$, etiquetado como "sigmoides". Por la superficie externa ambos se apreciaron dilatados y con una extensa zona de necrosis de aspecto hemorrágico en el ciego. Al abrirlo se apreciaron numerosas úlceras, de paredes irregulares de bordes edematosos y fondo amari-
Ilento, de tamaños variables de 0.5 hasta $1.3 \mathrm{~cm}$ de diámetro distribuidas a lo largo de ambos segmentos. Se identificaron dos grandes perforaciones en el ciego y en el sigmoides (Figura 1).

Histológicamente las úlceras tenían bordes limpios ligeramente elevados, de corte abrupto con la abertura luminal pequeña que se continuaba con un fondo amplio que semejaba la forma de un frasco de Erlenmeyer o del perfil de un botón de camisa o mancuernilla y que han sido ampliamente descritas como características de la colitis amebiana (Figuras 2 y 3). La necrosis del fondo de la úlcera estaba bien delimitada del infiltrado inflamatorio mixto y se identificaron numerosos trofozoítos de amibas redondeadas de aproximadamente 20 micras de diámetro de superficie externa, bien delineada, que resaltaba del tejido necrótico adyacente de citoplasma claro antófilo y finamente vacuolado con ocasional eritrofagocitosis (Figuras 4 y 5) que se tiñeron intensamente con tinción de PAS (ácido peryódico de Schiff) (Figura 6). Ante el hallazgo histológico mencionado se revisó nuevamente el apéndice encontrando el mismo tipo de parásitos menos bien conservados.

Aunque la amebiasis intestinal sigue estando presente en ciertas regiones de México las formas graves y complicadas, como la colitis ulcerada, el colon tóxico y el absceso hepático amebiano, son patologías muy poco frecuentes y rara vez reconocidas en la práctica médica actual. En el Cuadro 3 se presenta el resultado de una revisión de la casuística de 1971 a 2015, del Departamento de Patología del Instituto Nacional de Pediatría, que concentra los casos de amebiasis graves tanto quirúrgicos como de autopsia. De acuerdo con estas cifras podemos inferir que muchas generaciones de pediatras y cirujanos (y aún patólogos) hayan concluido con éxito su formación sin haber visto nunca un caso de amebiasis como el que ahora hemos presentado. 


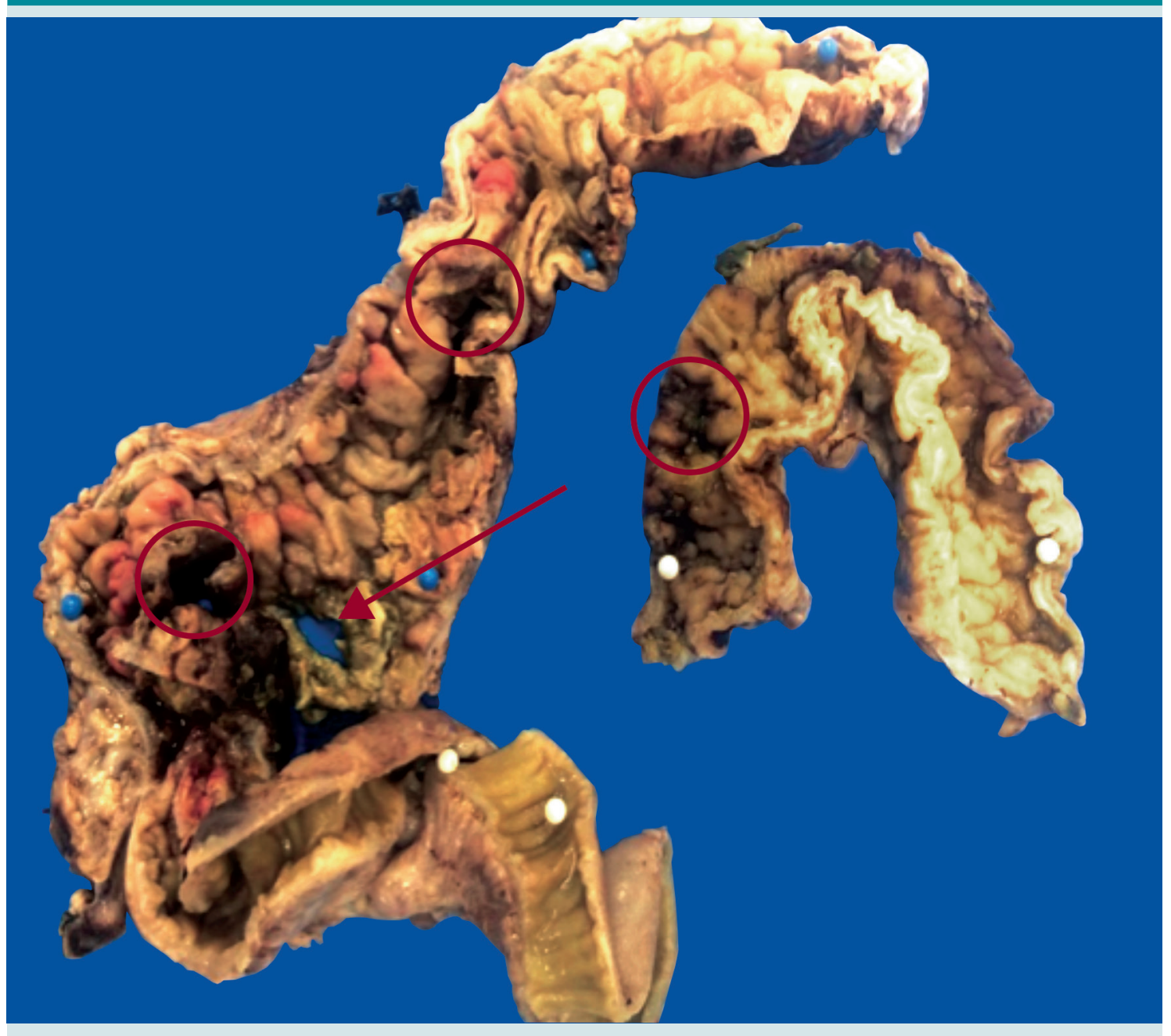

Figura 1. Producto de colectomía: segmento de íleon terminal, ciego y colon ascendente y fragmento de sigmoides. Se muestra la superficie mucosa con numerosas úlceras de contornos irregulares (círculos) y una perforación (flecha).

\section{DIAGNÓSTICOS ANATÓMICOS}

\section{AMIBIASIS INTESTINAL:}

1. Apendicitis aguda perforada con necrosis transmural y vascultis, peritonitis aguda purulenta.

2. Colitis aguda ulcerada y perforada con peritonitis.

\section{COMENTARIO DE PARASITOLOGÍA}

\section{Dra. Teresita Campos Rivera}

México continúa siendo un país endémico de amebiasis intestinal y por tanto aún es un problema de salud pública. Se considera que el número de portadores es del $20 \%$, enfermos $2 \%$, y muertes entre 0.1 y $0.2 \%$ al año. El $10 \%$ 
Ridaura-Sanz et al. Colitis ulcerada en preescolares

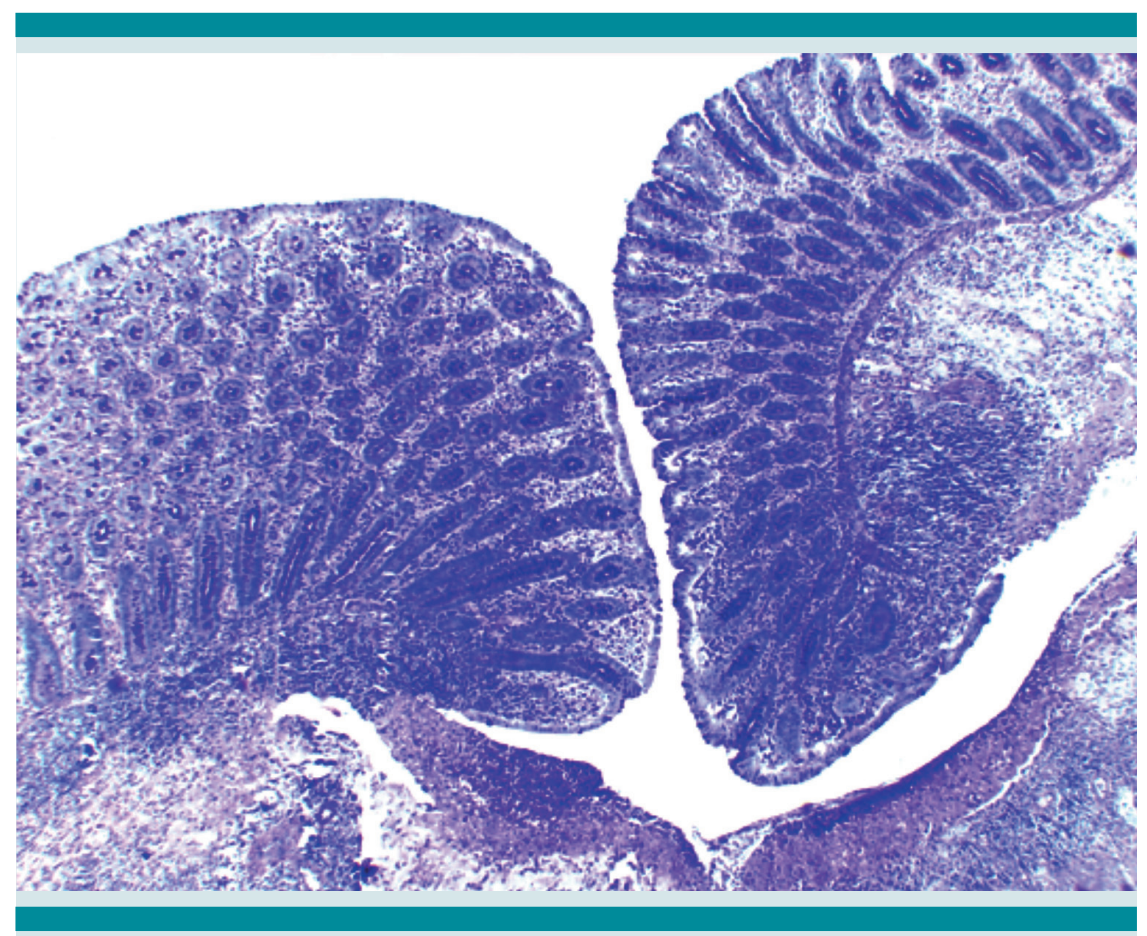

Figura 2. Imagen microscópica de una de las úlceras que muestra los bordes nítidos con la boca estrecha y fondo amplio (HE 4x).

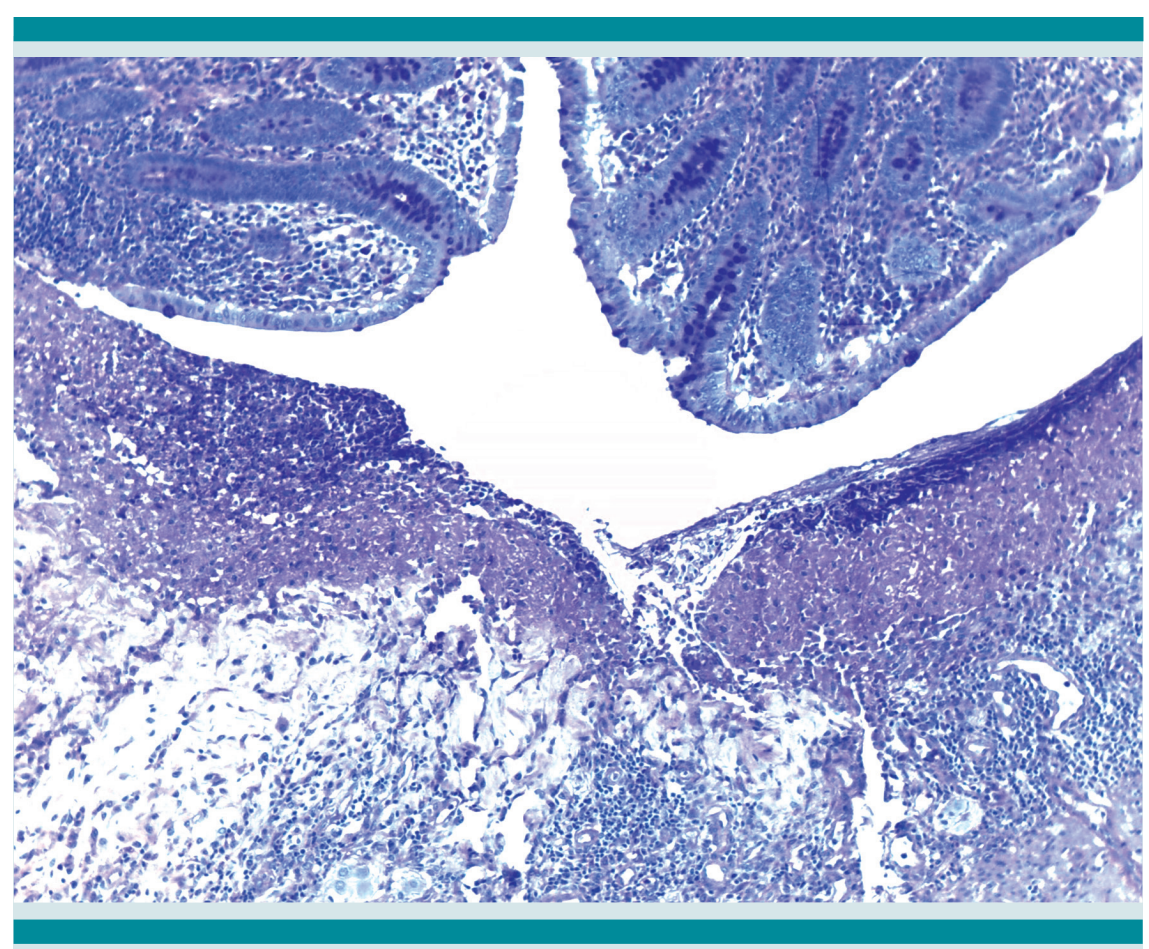

Figura 3. Fondo de la úlcera que muestra necrosis eosinofílica y una banda de infiltrado inflamatorio en la submucosa (HE 10x). 
Cuadro 3. Amebiasis: experiencia en el Departamento de Patología (INP, frecuencia 1971-2015)

\section{1 casos en autopsias}

$\begin{array}{ll}\text { Intestinal } & 11 \\ \text { Absceso hepático } & 12 \\ \text { Ambas } & 8 \\ \text { Último caso en } 1986 \text { (hace } 30 \text { años) } & \\ 24 \text { casos en material quirúrgico } & \\ \text { Intestinal } & 13 \\ \text { Apendicitis } & 5 \\ \text { Absceso hepático } & 5 \\ \text { Ambas } & 1 \\ \text { Último caso en } 2000 \text { (hace } 15 \text { años) } & \end{array}$

de los portadores desarrollará enfermedad; los portadores, al eliminar de forma espontánea al parásito ocasionan su prevalencia en el ambiente y su transmisión.

Los factores que favorecen el estado de portador son malos hábitos de higiene, ausencia de lavado de manos, inadecuada eliminación de excretas, contaminación de agua y alimentos con quistes de Entamoeba histolytica, presencia de vectores; todos estos factores se ven favorecidos por la falta de servicios, pobreza e ignorancia.

En el período 1995 a 2006 en México se estimó una tasa de morbilidad de amebiasis intestinal entre 1000 y 5000 casos por cada 100,000 habitantes. Datos epidemiológicos publicados por la Dirección General de Epidemiología para el año 2016 indican que la incidencia de amebiasis intestinal aguda fue de 220,205 casos, por lo que se considera dentro de las 20 principales causas de enfermedad nacional. La edad pediátrica más afectada fue de 1 a 4 años con 39,009 casos seguida por el grupo de 5 a 9 años con un total de 32,855 casos (Dirección General de Epidemiología, Anuario de morbilidad 1984-2016).

La virulencia de la E. histolytica va a depender de: 1) la capacidad infecciosa para colonizar el intestino y 2) de la capacidad de invasión; esto es, de diseminarse y destruir tejidos del huésped. Está virulencia se debe a la capacidad que tiene de producir lectinas que le permiten adherirse a las células y producir su lisis a través de las enzimas o proteinasas que degradan la elastina, el colágeno y la matriz extracelular.

El abordaje diagnóstico de la amebiasis intestinal aguda se lleva a cabo mediante un interrogatorio dirigido investigando los antecedentes epidemiológicos; son datos básicos que no se deben obviar. Hay que tener en cuenta que en nuestro país una gran parte de la población carece de servicios básicos en su vivienda como drenaje y agua potable, donde las condiciones de higiene no son las adecuadas. Hay que evaluar el cuadro clínico que se caracteriza por evacuaciones diarreicas con presencia de moco, sangre, pujo y tenesmo, así como dolor abdominal tipo cólico, con lo cual se integra la sospecha diagnóstica.

Para establecer el diagnóstico de amebiasis intestinal aguda se debe solicitar estudio microscópico de la materia fecal de evacuación líquida; este debe ser seriado para mejorar la sensibilidad (la muestra debe ser enviada al laboratorio inmediatamente después de obtenerse, se debe examinar entre 20 y 30 minutos después de su recolección para poder observar trofozoítos móviles, de lo contrario se pueden obtener resultados falsos negativos); se requiere por tanto de examen microscópico en fresco. Como complemento puede realizarse frote de materia fecal con tinción de Gömöri o hematoxilina férrica, en la que se deben observar trofozoítos, con lo cual se establece el diagnóstico. Esta es la forma directa y más rápida de establecer el diagnóstico, por lo que se requiere de personal capacitado. Otro estudio que es de utilidad es la detección de antígeno en heces.

En el caso de la amebiasis intestinal aguda no es necesario realizar examen coproparasitoscópico (CPS) de concentración flotación (Faust) seriado 


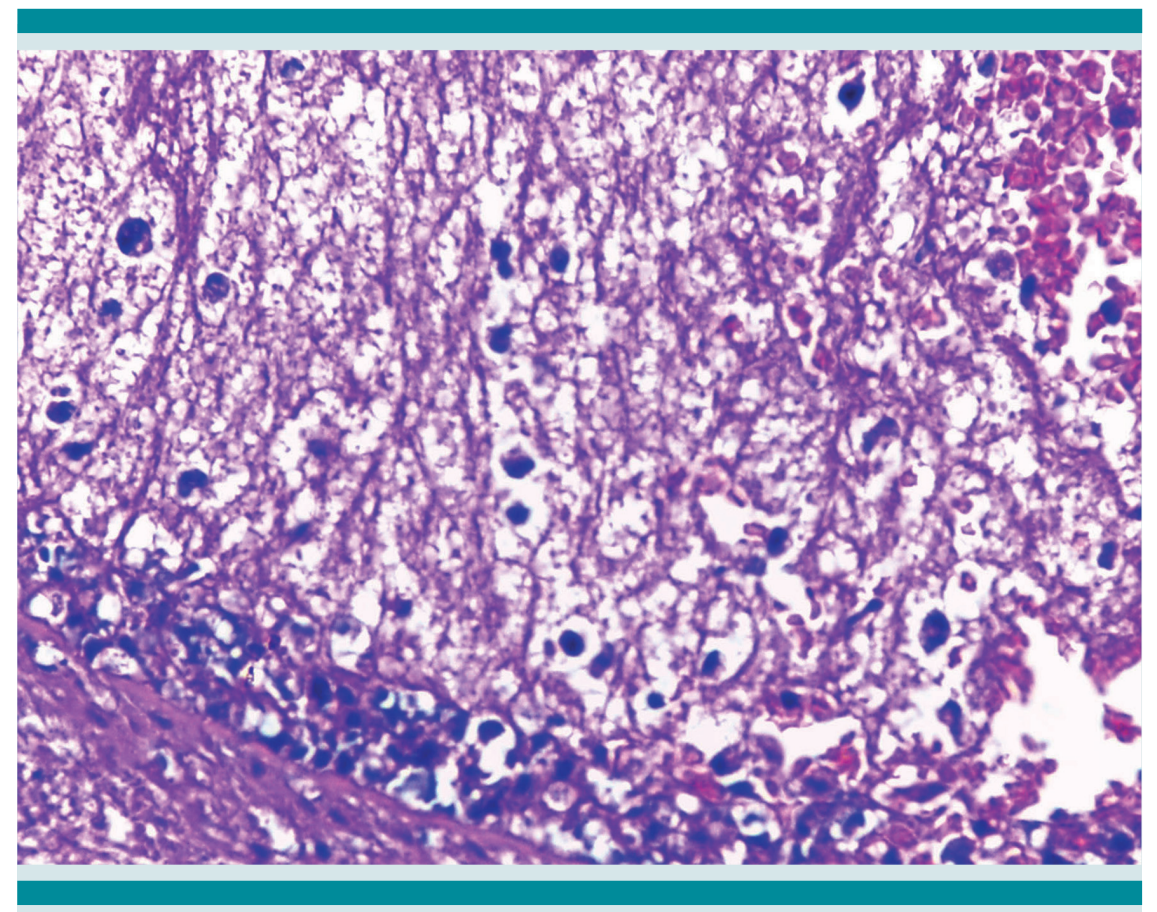

Figura 4. Fondo necrótico acelular que contiene numerosas estructuras redondas de límites precisos rodeados de un halo claro. Nótese la ausencia de elementos inflamatorios (HE 40x).

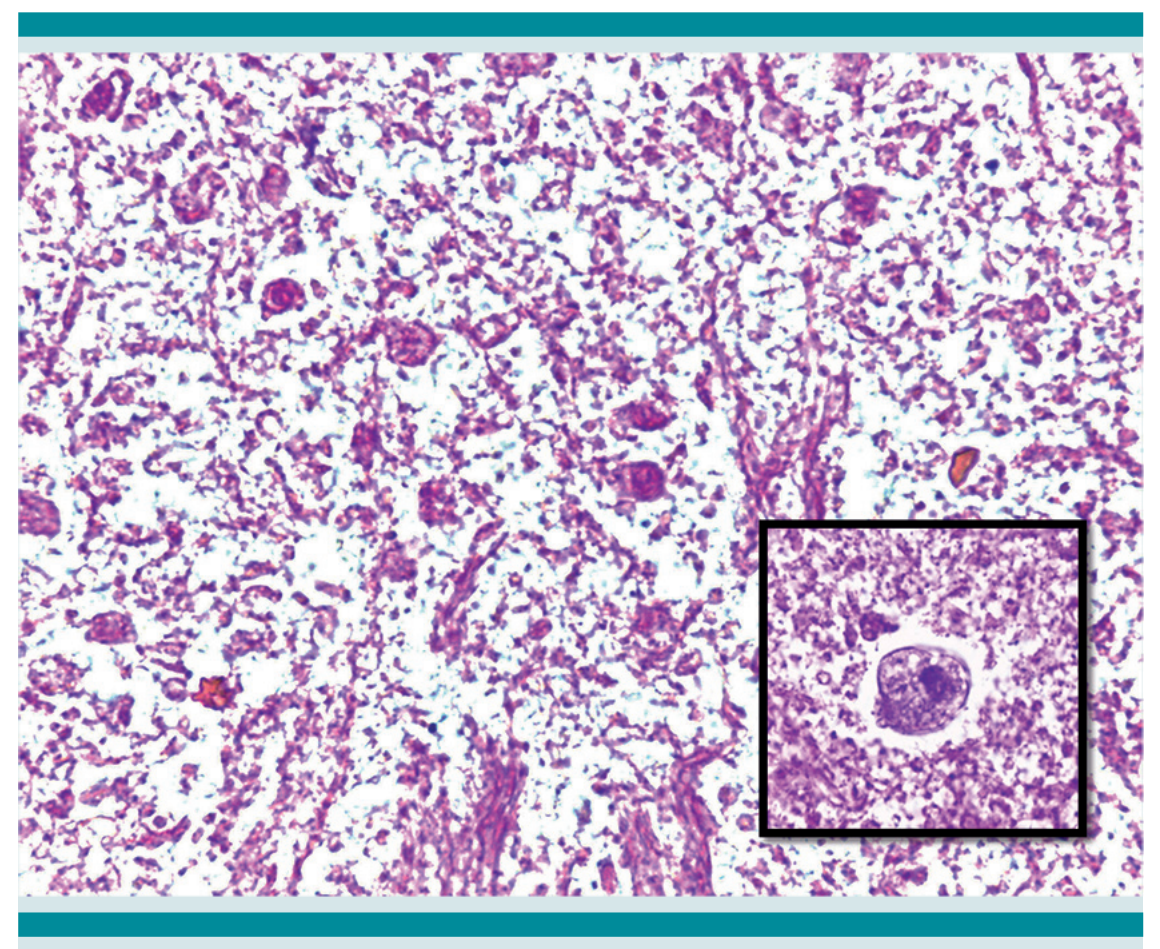

Figura 5. Trofozoítos en la zona de necrosis. Inserto: trofozoíto de E. histolytica con citoplasma vacuolado, membrana prominente y núcleo denso pequeño (HE 40x). 


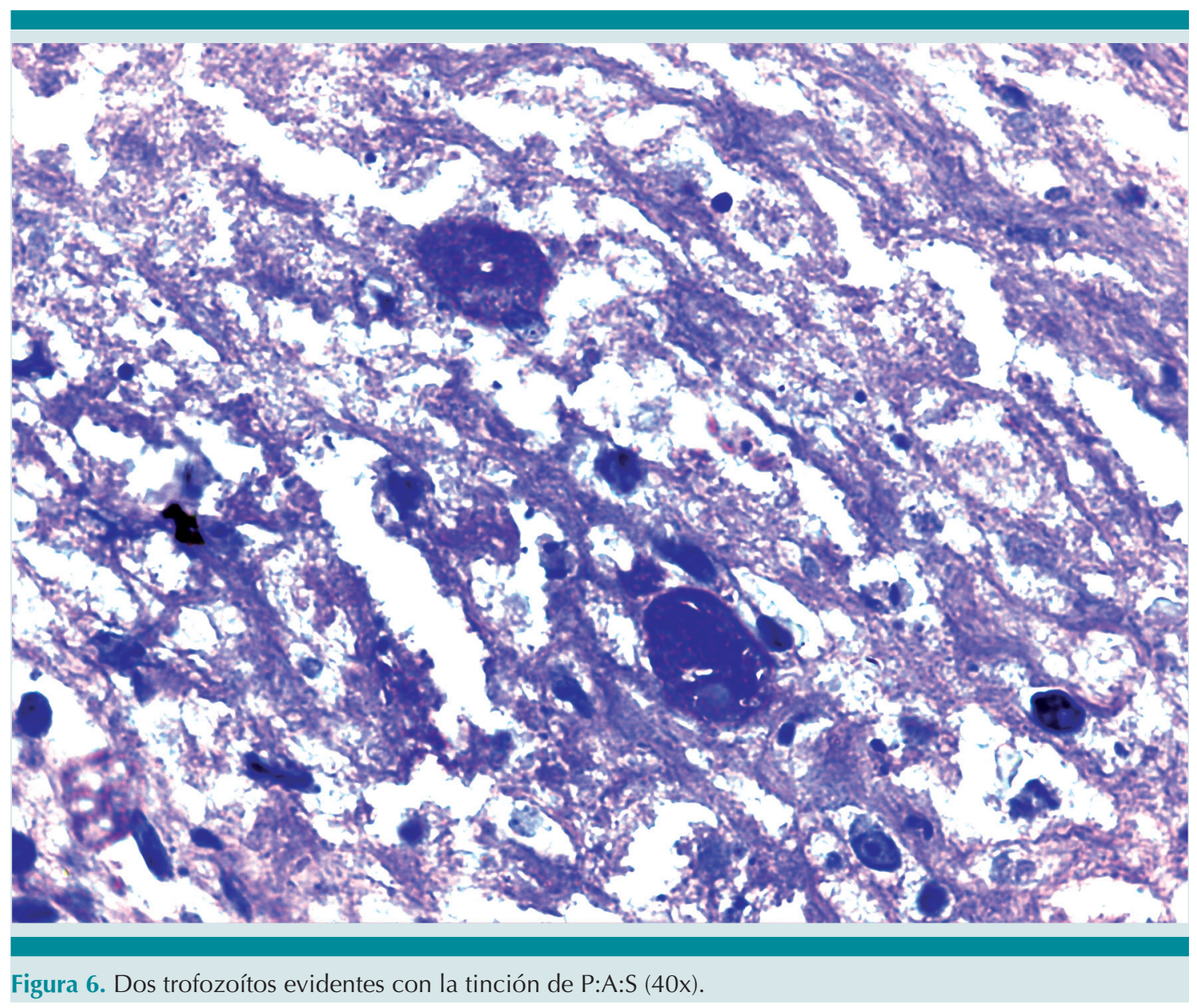

en tres muestras, ya que los trofozoítos sólo se van a encontrar en las evacuaciones líquidas recién emitidas, el CPS sirve para identificar quistes que se presentan en la forma crónica o en portadores asintomáticos.

Las técnicas indirectas de diagnóstico, como la detección de anticuerpos específicos, son de utilidad para la amebiasis invasiva (fijación del complemento, hemaglutinación indirecta, entre otras). Es importante mencionar que los anticuerpos solo aparecen cuando hay invasión a los tejidos y persisten elevados durante algún tiempo. En relación con las pruebas mencionadas se consideran títulos altos por arriba de
$1: 128$ y $1: 512$, respectivamente, pero en los casos de absceso hepático los títulos suelen ser más elevados con ambas pruebas.

En nuestra experiencia el tratamiento de primera elección sigue siendo el metronidazol a dosis de $30 \mathrm{mg} / \mathrm{kg} / \mathrm{día}$, fraccionado en 3 dosis durante 10 días vía oral; los pacientes que no completan el tiempo de tratamiento presentan recaídas de amebiasis intestinal aguda meses después de haber abandonado el tratamiento con metronidazol. Con este medicamento se tiene una eficacia que va de 90 a $96 \%$. Por lo anterior, se recomienda después de concluido el esquema hay que descolonizar a E. histolytica de 
la luz intestinal mediante la administración de un antiamebiano de acción intraluminal como la diyodohidroxiquinoleína en dosis de $30 \mathrm{mg} / \mathrm{kg} /$ día en niños, fraccionada en 3 dosis y administrada durante 10 días V.O. Otros medicamentos que se pueden utilizar son tinidazol, que ha mostrado eficacia de 76 a 96\% o nitazoxanida con eficacia de $75 \%$.

Se recomienda que después de concluido el tratamiento, y de la desaparición del cuadro diarreico, se deberá realizar control posterapéutico dos semanas después, mediante la realización de examen CPS de concentración flotación seriado en tres muestras. Si se reporta nuevamente la presencia del protozoario se deberá dar tratamiento con un antiamebiano de acción intraluminal como la diyodohidroxiquinoleína.

\section{LECTURAS RECOMENDADAS}

1. Haque $R$, Huston $C D$, Hughes $M$, Houpt $E$, Petri Jr. WS. Amebiasis. N Eng J Med 2003,348:1565-73.

2. Ximénez C, Morán P. Ramos F., Ramiro M. Amibiasis intestinal: estado actual del conocimiento. Med Int Mex. 2007;23 (5):398-407.

3. Olivos García A, Saavedra E, Nequiz Avendaño N, Pérez Tamayo R. Amibiasis: mecanismos moleculares de la patogenicidad de Entaboeba histolytica. Rev Fac Med UNAM. 2011; 54:10-20.

4. Ximénez $C$, Morán $P$, Rojas $L$, Valadez A, Gómez A, Ramiro M, Cerritos R, González E, Hernández E, Oswaldo P. Novelties on ameobiasis: A neglected tropical disease. J Glob Infect Dis. 2011;3(2):166-174.

5. Rojas L, Morán P, Valadez A, Gómez A, González E, Hernández E, Partida O, Nieves M, Gudiño M, Magaña U, Torres J, Ximénez. Entamoeba histolytica and Entamoeba dispar infection in Mexican school children: genotyping and phylogenetic relationship. BMC Infectious Dis. 2016;16:485. DOI 10.1186/s12879-016-1812-8

6. Vázquez TO. Amibiasis Intestinal Aguda en Guía de tratamiento de las Enfermedades Parasitarias. Edit. Trillas 2009 ISBN 978-607-17-0079-7 p.15-17.

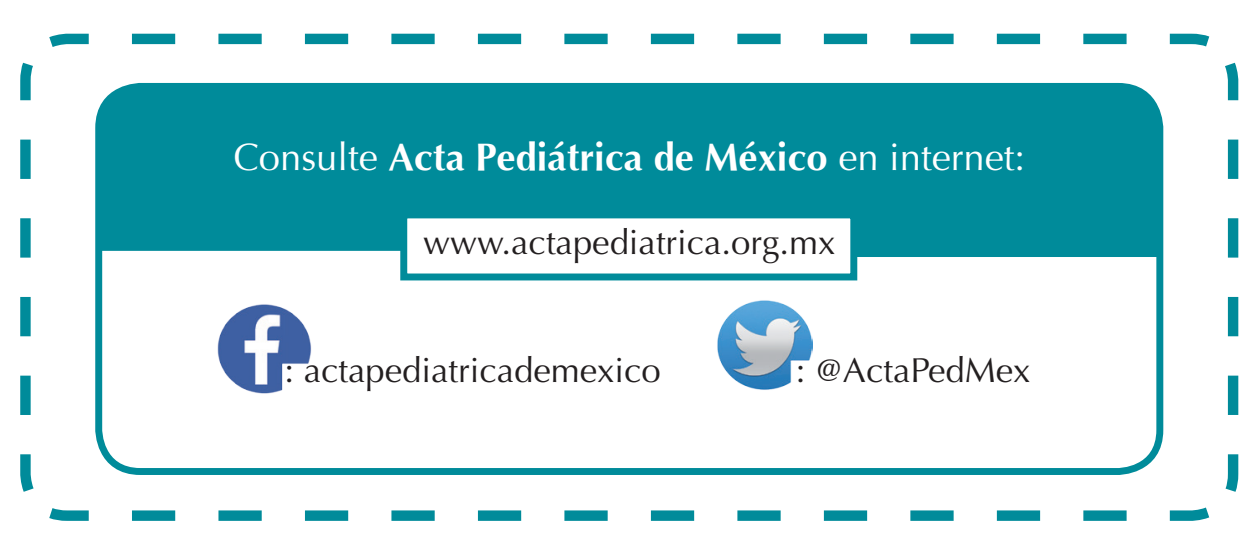

